\title{
Evaluation of Matrix Band Systems for Posterior Proximal Restorations among Egyptian Dentists: A Cross-Sectional
}

\section{Survey}

\section{Evaluacija sustava trakastih dentalnih matrica stražnjih zuba među egipatskim stomatolozima: presječna anketa}

Cairo University, Faculty of Dentistry, Conservative Dentistry Department, Egypt

Zavod za restaurativnu stomatologiju Stomatološkog fakulteta Sveučilišta u Kairu, Egipat

\section{Abstract}

Objectives: This study aimed to investigate the techniques of matricing used by Egyptian dentists, and evaluate the influence of these techniques on the reproduction of optimum proximal contacts for posterior proximal resin composite restorations. Material and methods: An online questionnaire was developed and sent to 785 dentists via e-mail and social media platforms. The survey asked the participants about the following: the highest academic degree achieved and their experience, techniques of matricing used, brands of matricing systems used, assessment of proximal contact points, their evaluation of the contact points they reproduced, and their assessment of the restorations' emergence profiles. Results: A total of 415 dentists participated in the study (response rate $52.8 \%$ ). 308 dentists (74\%) preferred using the sectional matrix system, while 107 dentists $(26 \%)$ preferred using the circumferential matrix system. One hundred twenty-six dentists (31\%) reported that the circumferential matrix systems reproduced optimum contacts, 105 dentists $(25 \%)$ reported tight contacts and 184 dentists (44\%) reported open contacts. However, for the sectional matrix systems, the optimum contacts were reported by 279 dentists (67\%) tight contacts by 109 dentists (26\%) and open contacts by 27 dentists ( $7 \%$ ). There was a statistically significant difference between the sectional matrix systems and the circumferential matrix systems regarding the tightness of the proximal contact points $(P<0.0001)$. Conclusions: Egyptian dentists preferred using the sectional matrix systems. The survey indicated that optimum contact points were highly associated with the sectional matrix systems, while poor (open and tight) contacts were highly associated with the circumferential matrix systems.
Received: August 3rd, 2020

Accepted: November 2nd, 2020

Address for correspondence Omar Osama Shaalan, PhD Faculty of Dentistry

Conservative Dentistry Department Cairo University, Cairo, Egypt. 35 Mohamad Farid Street El Hay El Motamayz,

Phone: (002)01122665661,

Fax: (002) 38831188, omar.shaalan@dentistry.cu.edu.eg

Key words

Circumferential; Class II; Matrix bands; Proximal; Resin composite; Sectional

\section{Introduction}

Proximal contact points between the teeth protect the integrity of the stomatognathic system by providing a balance against the anterior component of the force created by the tendency of teeth for mesial drifting (1). Failure to recreate contact points will break the equilibrium and harmony, and the remaining components of the stomatognathic system will spontaneously try to establish a new equilibrium(2).

A great challenge for dentists is to reproduce optimum contact points while trying to achieve clinically well-accepted restorations, especially with resin composite materials. Proximal restorations should compensate for the thickness of the matrix band, and for inevitable polymerization shrinkage of the resin restorative material. Numerous techniques have been implemented to achieve optimum contact points with proximal resin composite restorations (3). In this respect, the selection of a proper matrix system and separation technique is a significant factor. In the past, traditional circumferential

\section{Uvod}

Aproksimalne kontaktne točke između zuba štite integritet stomatognatnog sustava pružanjem ravnoteže prednjoj komponenti sile koja se stvara tendencijom zuba da se mezijalno pomiču (1). Ako se ne rekonstruiraju adekvatne kontaktne točke, narušit će se ravnoteža i sklad, a preostale komponente žvačnog organa spontano će pokušati uspostaviti novu ravnotežu (2).

Za kliničara je velik izazov rekonstruirati optimalne kontaktne točke dok pokušava postići klinički prihvatljive ispune, posebno s kompozitnim materijalima. Aproksimalni ispuni trebali bi kompenzirati debljinu interdentalne matrice i neizbježnu polimerizacijsku kontrakciju smolastoga restaurativnog materijala. Mnogobrojnim se tehnikama postižu optimalne kontaktne točke s aproksimalnim ispunima od kompozita (3). Pritom je važan čimbenik odabir odgovarajućega matričnog sustava i tehnike separacije. U prošlosti su najčešće korištene matrice bili tradicionalni obuhvatni matrični susta- 
matrix systems were the most common matrices used by dentists, although they exhibited limitations in reproducing correct proximal contact points, and improper flat matrix contour (4).

The introduction of the separation ring and pre-contoured sectional matrix systems had a substantial influence on the tightness of the resultant proximal contacts of dental restorations, along with a consistent contact tightness (2-4). However, the reliability and repeatability of the recreation of optimum, anatomically correct proximal contact points can sometimes be unpredictable. Thus, dental practitioners must develop the essential skills, gain the necessary experience and acquire an appropriate armamentarium to provide optimum oral health care for their patients, to be able to restore missing surfaces of teeth back to normal function and esthetics without compromising any biological or mechanical aspect (5). Based on the above-mentioned data, the current cross-sectional survey aimed to investigate the techniques of matricing used by Egyptian dentists and evaluate the influence of these techniques on the reproduction of optimum proximal contacts for posterior proximal resin composite restorations.

\section{Material and Methods}

An online questionnaire was developed by a staff member at the Faculty of Dentistry, Cairo University, via Google forms. It was sent to 785 dentists who were representing faculties of dentistry among the Greater Cairo Governate through the period of one month, between the $8^{\text {th }}$ of June and the $8^{\text {th }}$ of July 2020 , either via e-mail or social media platforms (Facebook and WhatsApp, San Francisco, California, USA). The questionnaire included eleven questions asking the participants about their highest academic degree achieved, experience, techniques of matricing used, brands of matricing systems used, assessment of proximal contact points, their evaluation of the contact points they reproduced and their assessment of the emergence profiles of restorations (Table 1). Proximal contacts were categorized as optimum, open and tight. If dental floss passed through the contact area as natural dentition on the other side, it was considered as optimum contact point. Open contact points were considered when dental floss passed through the contact area without resistance, while proximal contact was considered as tight when dental floss could not be passed at all or was torn (6).

The sample size was calculated using StatCalc version 1.4.3 (Epi Info ${ }^{\mathrm{TM}}$, CDC, Atlanta, Georgia, USA) using the random sampling test, implementing 120000 population size, $95 \%$ of confidence level and $5 \%$ of confidence limits. To be able to represent the total dentist population, the calculated sample size was a total of 383 participants. Data were collected anonymously from Google forms.

\section{Statistical analysis}

Statistical analysis was performed using the MedCalc software (MedCalc Software bvba) version 19 for Windows. Data were analyzed using descriptive statistics and represented as frequency and percentage in pie charts. The Chi-square test was used to compare the tightness of proximal contact points reported by participants, either for circumferential vi, no unatoč tomu pokazivali su ograničenja u reprodukciji adekvatnih aproksimalnih kontaktnih točaka zbog ravne konture matrice (4).

Kada su se počeli upotrebljavati separacijski prstenovi i unaprijed oblikovan sekcijski matrični sustav, to je imalo golem utjecaj na tjesnoću nastalih aproksimalnih kontakata ispuna $(2-4)$. No pouzdanost i ponovljivost rekonstrukcije optimalnih, anatomski pravilnih aproksimalnih kontaktnih točaka katkad može biti nepredvidiva. Zato kliničari moraju svladati osnovne vještine, steći potrebno iskustvo i imati odgovarajuća sredstva za pružanje optimalne oralne zdravstvene zaštite svojim pacijentima kako bi mogli rekonstruirati izgubljene površine zuba, te vratiti normalnu funkciju i estetiku a da ne ugroze biološke ili mehaničke aspekte (5). Na temelju spomenutih podataka, cilj ove presječne ankete bio je istražiti sustave interdentalnih matrica koje upotrebljavaju egipatski stomatolozi i procijeniti njihov utjecaj na rekonstrukciju optimalnih aproksimalnih kontakata na stražnjim aproksimalnim kompozitnim ispunima.

\section{Materijal i metode}

$\mathrm{Na}$ Stomatološkom fakultetu Sveučilišta u Kairu pripremljen je upitnik za ovo istraživanje putem Googleovih obrazaca. Poslan je na 785 adresa dentalnih doktora, koji su predstavljali stomatološke fakultete na širem području Kaira, u roku od mjesec dana između 8. lipnja i 8. srpnja 2020., bilo e-poštom bilo putem platformi društvenih medija (Facebook i WhatsApp, San Francisco, Kalifornija, SAD). Upitnik je sadržavao jedanaest pitanja o najvišem postignutom akademskom stupnju sudionika, iskustvu, korištenim sustavima interdentalnih matrica, markama korišstenih sustava, procjeni kontaktnih točaka, procjeni kontaktnih točaka koje su oni rekonstruirali i o njihovoj procjeni izlaznih profila restauracija (tablica 1.). Aproksimalni kontakti kategorizirani su kao optimalni, otvoreni i tijesni. Ako je zubni konac prolazio kroz kontaktno područje kao na prirodnoj denticiji s druge strane, kontaktna točka smatrala se optimalnom. Otvorene kontaktne točke postojale su ako je zubni konac prolazio kroz kontaktno područje bez otpora, a ako uopće nije mogao proći ili je puknuo, aproksimalni kontakt smatrao se tijesnim (6).

Veličina uzorka izračunata je u programu StatCalc, verzija 1.4.3 (Epi InfoTM, CDC, Atlanta, Georgia, SAD) korištenjem testa slučajnog uzorkovanja, primjenom veličine populacije od 120000 , razine pouzdanosti od $95 \%$ i granice pouzdanosti od $5 \%$. Da bi se mogla reprezentirati ukupna populacija stomatologa, izračunata veličina uzorka iznosila je ukupno 383 sudionika. Podatci su prikupljeni anonimno s Googleovih obrazaca.

\section{Statistička analiza}

Statistička analiza obavljena je u softveru MedCalc (MedCalc Software bvba), verzija 19 za Windowse. Podatci su analizirani deskriptivnom statistikom i predstavljeni kao učestalost i postotak u obliku tortnih grafikona. Hi-kvadrat test upotrijebljen je za usporedbu nepropusnosti aproksimalnih kontaktnih točaka koje su sudionici prijavili, bilo za obu- 
Table 1 Questionnaire

Tablica 1. Upitnik

Questions • Pitanja

Answers • Odgovori

a- What is your highest academic degree? $\bullet$ Koji je vaš najviši akademski stupanj?

1. BDS • prvostupnik

2. $\mathrm{MSc} \cdot$ magistar

3. $\mathrm{DDS} / \mathrm{PhD} \cdot$ doktor znanosti

4. Egyptian fellowship board $\bullet$ Egipatski odbor

b- Which technique of matricing do you prefer to

1. Circumferential $\bullet$ obuhvatni

use? $\bullet$ Koji sustav interdentalnih matrica preferirate? 2 2. Sectional $\bullet$ sekcijski

c- Which technique is the easiest to apply? $\bullet$ Koju je 1 . Circumferential $\bullet$ obuhvatnu

tehniku najjednostavnije primijeniti?

2. Sectional $\bullet$ sekcijsku

d- If you use circumferential matricing, which holder do you use? • Ako upotrebljavate obuhvatnu matricu, kojim se držačem koristite?

1. Tofflemire (not branded). $\bullet$ Tofflemire (nije brendiran)

2. Ivory no. 2 (not branded). $\bullet$ Ivory br. 2 (nije brendiran)

3. Ivory no. 8 (not branded). $\bullet$ Ivory br. 8 (nije brendiran)

4. Automatrix, Dentsply Sirona, Konstanz, Germany. • Automatrix, Dentsply Sirona, Konstanz, Njemačka

5. Do not use $\bullet$ Ne upotrebljavam

1. Classic ring and pre-contoured matrix, TOR VM, Moscow, Russia. $\bullet$ Klasičnom i unaprijed oblikovanom matricom TOR VM, Moskva, Rusija

2. Delta ring and pre-contoured matrix, TOR VM, Moscow, Russia. • Prstenom Delta i unaprijed oblikovanom matricom TOR VM, Moskva, Rusija

3. MD ring and pre-contoured matrix, TOR VM, Moscow, Russia. • Prstenom MD i unaprijed oblikovanom matricom TOR VM, Moskva, Rusija

4. Saddle holder and pre-contoured matrix, TOR VM, Moscow, Russia. • Sedlastim držačem i unaprijed oblikovanom matricom TOR VM, Moskva, Rusija

e- If you use sectional matrix, which system do you use? • Ako upotrebljavate sekcijsku matricu, kojim se sustavom koristite?

5. Composi-Tight 3DFusion and pre-contoured matrix, Garrison Dental Solutions, Spring Lake, Michigan, USA. • Composi-Tight 3Dfusionom i unaprijed oblikovanom matricom Garrison Dental Solutions, Spring Lake, Michigan, SAD

6. Palodent V3 ring and pre-contoured matrix, Dentsply Sirona, Konstanz, Germany. Prstenom Palodent V3 i unaprijed oblikovanom matricom Dentsply Sirona, Konstanz, Njemačka

7. Ena ring and pre-contoured matrix, Micerium, Italy. Prstenom Ena i unaprijed oblikovanom matricom Micerium, Italija

8. Twin ring and pre-contoured matrix, Bioclear, Tacoma/WA, USA. • Prstenom Twin i unaprijed oblikovanom matricom Bioclear, Tacoma/WA, SAD

9. Do not use $\bullet$ Ne upotrebljavam

10. Other. $\bullet$ Ostalo

1. Wooden wedges (not branded). $\bullet$ Drveni (nije brendiran)

2. Diamond, Bioclear, Tacoma/WA, USA. • Dijamantni, Bioclear, Tacoma/WA, SAD

f- Which kind of wedge do you use? • Koju vrstu interdentalnog kolčića upotrebljavate?

3. Elastic wedges, TOR VM, Moscow, Russia. • Elastični, TOR VM, Moskva, Rusija

4. Plastic wedges, TOR VM, Moscow, Russia. • Plastični, TOR VM, Moskva, Rusija

5. Other. $\bullet$ Ostalu

g- Do you use dental floss to check contact tightness after restoration? • Upotrebljavate li zubni konac da biste provjerili tjesnoću kontaktne točke?

\section{Yes $\bullet \mathrm{Da}$}

2. $\mathrm{No} \bullet \mathrm{Ne}$

1. Optimum: Dental floss pass through contact area as natural dentition on the other side $\bullet$ Optimalna: zubni konac prolazi kroz kontaktno područje kao na prirodnoj denticiji s druge strane

h- When you use circumferential matrix systems, describe tightness of the contact produced? • Ako upotrebljavate obuhvatne matrične sustave, opišite tjesnoću stvorenog kontakta?

2. Open: Dental floss pass without resistance. • Otvorena: zubni konac prolazi bez otpora

3. Tight: Dental floss could not be passed at all or was torn. $\bullet$ Tijesna: zubni konac ne može proći ili pukne

i- Describe emergence profile (cervical outline of restoration as it emerges from periodontium) produced with circumferential matrix $\bullet$ Opišite izlazni profil nicanja (cervikalni obris restauracije kad izlazi iz parodonta) izrađen obuhvatnom matricom

j- When you use sectional matrix systems, describe tightness of the contact produced? • Ako upotrebljavate sekcijske matrične sustave, opišite tjesnoću nastalog kontakta?

1. Proper $\bullet$ Pravilan

2. Improper $\bullet$ Nepravilan

k. Describe emergence profile (cervical outline of restoration as it emerges from periodontium) produced with sectional matrix $\bullet$ Opišite izlazni profil nicanja (cervikalni obris restauracije kad izlazi

1. Optimum: Dental floss pass through contact area as natural dentition on the other side $\bullet$ Optimalna: zubni konac prolazi kroz kontaktno područje kao na prirodnoj denticiji s druge strane

2. Open: Dental floss pass without resistance. $\bullet$ Otvorena: zubni konac prolazi bez otpora

3. Tight: Dental floss could not be passed at all or was torn. $\bullet$ Tijesna: zubni konac ne može proći ili pukne

1. Proper $\bullet$ Pravilan

2. Improper $\bullet$ Nepravilan

iz parodonta) izrađen sekcijskom matricom 
matrix systems or sectional matrix systems. A P value $\leq 0.05$ was considered statistically significant.

\section{Results}

A total of 415 dentists participated in the current survey with a response rate of $52.8 \%$. Figure 1 is a pie chart representing the results of the questionnaire. Two hundred fifteen dentists (52\%) were Bachelor of Dental Surgery (BDS) holders, 136 (33\%) were Master of Science (MSc) holders, 44 (10\%) were Doctor of Dental Sciences/ Doctor of Philosophy (DDSc/PhD) holders and 20 dentists (5\%),were holders of the Egyptian Fellowship Board of Dentistry. Three hundred eight dentists (74\%) preferred using the sectional matrix system, while 107 dentists (26\%) preferred using the circumferential matrix system. Two hundred sixty-three dentists (63\%) selected the sectional matrix systems as easily used and placed, while 152 dentists (37\%) selected the circumferential matrix systems as easily used and placed. Regarding the dentists who used the circumferential matrix systems, 333 dentists $(80.2 \%)$ used the Tofflemire holder, while 19 dentists (4.6\%) used the Ivory no. 2 holder, 8 dentists (2\%) used the Ivory no. 8 holder, and 3 dentists $(0.7 \%)$ used the Automatrix holder (Dentsply Sirona, Konstanz, Germany). Fifty-two dentists (12.5\%) reported not using the circumferential matrix systems at all, and they used only the sectional matrix systems.

Concerning the dentists who used the sectional matrix systems, 95 dentists (22.9\%) used the classic ring system (TOR VM, Moscow, Russia) 86 dentists (20.7\%) used the Composi-Tight system (Garrison Dental Solutions, Spring Lake, MI, USA), 68 dentists (16.4\%) used the delta ring system (TOR VM, Moscow, Russia) 63 dentists (15.2\%) used the Palodent V3 system (Dentsply Sirona, Konstanz, Germany) 26 dentists (6.3\%) used the MD ring system (TOR VM, Moscow, Russia) 12 dentists (2.9\%) used the Ena ring system (Micerium, Avegno, Genoa, Italy) 4 dentists (1\%) used the saddle system (TOR VM, Moscow, Russia) and 3 dentists $(0.7 \%)$ used the Twin ring system (Bioclear, Tacoma, WA, USA). Fifty-eight dentists (13.9\%) reported not using the sectional matrix systems at all, and they used only the circumferential matrix systems. Two hundred forty-six dentists (59\%) used wooden wedges, while 75 dentists $(18 \%)$ used diamond wedges, 68 dentists $(17 \%$,) used plastic wedges and 26 dentists $(6 \%)$ used elastic wedges. Three hundred thirtyeight dentists out of 415 dentists (81\%) checked the tightness of proximal contact after restorations, while 77 dentists (19\%) did not check the tightness of proximal contacts.

One hundred twenty-six dentists (31\%) reported optimum contacts, 105 dentists $(25 \%)$ reported tight contacts and 184 dentists (44\%) reported open contacts with the circumferential matrix systems. The improper emergence profile of proximal contour was reported by 348 dentists (95.8\%). However, regarding the sectional matrix systems, optimum contacts were reported by 279 dentists $(67 \%)$ tight contacts by 109 dentists $(26 \%)$ and open contacts by 27 dentists $(7 \%)$. The proper emergence profile of proximal contour was reported by 347 dentists (97.2\%). There was a statisti- hvatne bilo za sekcijske matrične sustave. Vrijednost $\mathrm{P} \leq 0,05$ smatrala se statistički značajnom.

\section{Rezultati}

Ukupno 415 stomatologa sudjelovalo je u ovom istraživanju sa stopom odgovora od 52,8 \%. Na slici 1 . je tortni grafikon koji prikazuje rezultate dobivene upitnikom. Dvjesto petnaest (52\%) stomatologa bili su prvostupnici stomatološke kirurgije (BDS), 136 (33\%) magistri, 44 (10\%) doktori znanosti i $20(5 \%)$ članovi egipatskoga Odbora za stomatologiju. Tristo osam (74\%) stomatologa radije je upotrebljavalo sustav sekcijskih matrica, a $107(26 \%)$ preferiralo je obuhvatne matrične sustave. Dvjesto šezdeset i tri $(63 \%)$ stomatologa odabrala su sekcijske matrične sustave kao jednostavne za upotrebu i postavljanje, a $152(37 \%)$ obuhvatne. Kad je riječ o stomatolozima koji su se koristili obuhvatnim matričnim sustavima, njih $333(80,2 \%)$ upotrebljavalo je držač Tofflemire, $19(4,6 \%)$ držač br. 2 Ivory, osam (2\%) držač br. 8 i tri $(0,7 \%)$ držač Automatrix (Dentsply Sirona, Konstanz, Njemačka). Pedeset i dva (12,5\%) stomatologa izvijestila su da uopće ne upotrebljavaju obuhvatne matrične sustave, nego samo sekcijske matrice.

Tri stotine i osam (74 \%) stomatologa radije je upotrebljavalo sustav sekcijskih matrica, a $107(26 \%)$ preferiralo je obuhvatne matrične sustave. Dvije stotine šezdeset i tri $(63$ $\%)$ stomatologa odabrala su sekcijske matrične sustave jer se lako upotrebljavaju i postavljaju, a $152(37 \%)$ su se iz istog razloga odlučila za obuhvatne matrične sustave kao jednostavne za upotrebu i postavljanje. Među stomatolozima koji su se koristili obodnim matričnim sustavima, njih 333 (80,2 \%) odabrali su držač Tofflemire, a $19(4,6 \%)$ je upotrebljavalo držač br. 2 Ivory, 8 (2\%) držač br. 8 te $3(0,7 \%)$ rabljene držače Automatrix (Dentsply Sirona, Konstanz, Njemačka). Pedeset i dva (12,5\%) stomatologa izvijestila su da se uopće ne koriste obodnim matričnim sustavima nego samo sekcijskima.

Među stomatolozima koji su upotrebljavali sekcijske matrične sustave, njih $95(22,9 \%)$ koristilo se klasičnim prstenastim sustavom (TOR VM, Moskva, Rusija), 86 (20,7 \%) sustavom Composi-Tight (Garrison Dental Solutions, Spring Lake, MI, SAD), 68 (16,4\%) sustavom Delta prsten (TOR VM, Moskva, Rusija), 63 (15,2 \%) sustavom Palodent V3 (Dentsply Sirona, Konstanz, Njemačka), 26 (6,3\%) prstenastim sustavom MD (TOR VM, Moskva, Rusija), 12 (2,9 \%) prstenastim sustavom Ena (Micerium, Avegno, Genova, Italija), 4 (1\%) sedlastim sustavom (TOR VM, Moskva, Rusija) i $3(0,7 \%)$ sustavom Twin ring (Bioclear, Tacoma, WA, $\mathrm{SAD})$. Pedeset osam $(13,9 \%)$ stomatologa izvijestilo je da uopće ne upotrebljavaju sekcijske matrične sustave, nego samo obuhvatne. Dvije stotine četrdeset i šest (59 \%) stomatologa upotrebljavalo je drvene interdentalne kolčiće, 75 (18 $\%)$ dijamantne, $68(17 \%)$ plastične i $26(6 \%)$ elastične. Tri stotine trideset i osam stomatologa $(81 \%)$ od njih 415 provjeravalo je tjesnoću aproksimalnog kontakta nakon dovršavanja ispuna, a $77(19 \%)$ to nije činilo.

Stotinu dvadeset i šest (31\%) stomatologa prijavilo je optimalne kontakte, 105 (25\%) tijesne kontakte, a 184 (44\%) 
cally significant difference between the sectional matrix systems and the circumferential matrix systems regarding the tightness of the proximal contact points as reported by dentists $(\mathrm{P}<0.0001)$

\section{Discussion}

Mastering proximal tooth-colored restorations with resin composite is very critical. The success of posterior composite restorations is attributed to the operator's skills, the characteristics of the material as well as the placement techniques used (7). Numerous studies confirmed that pre-contoured sectional matrix bands provided the resin restorations with optimum contours and emergence profiles when compared to conventional flat circumferential matrix bands $(3,4)$. It has been confirmed by several studies that the use of precontoured sectional matrix bands in combination with a separation ring achieved superior contact tightness owing to the interdental separation exerted by the ring during restoration, as well as the contour of the matrix, which mimics the natural proximal contours and emergence profiles of the teeth $(3,8-10)$.

In the current study, most of the dentists did not believe that the circumferential matrix systems produce consistent contact points. The reason behind the less-than-optimal proximal contact with circumferential matrix systems might be attributed to the insufficient movement of adjacent teeth from wedge placement compared to the interdental separation ring and the flat matrix band. Tight contact points were reported at a higher level occlusally, as dental floss could not pass or was torn upon placement (11). Optimum contact points with circumferential matrix systems were mostly reported by some more experienced dentists (DDSc/PhD) which might be accredited to the long period of practice using the circumferential matrix systems. Nevertheless, they described the emergence profiles as improper (12).

The results of the current survey showed that $74 \%$ of the dentists preferred using the sectional matrix systems and $63 \%$ of the dentists considered the sectional matrix systems to be easily used and applied compared to the circumferential matrix systems. This may be attributed to the shift in dental restorations done by dentists from amalgam to resin composites, based on patient's esthetic demands and the development in physical and mechanical properties of resin composites, which requires the training on the new techniques and restorative procedures at dental schools. However, these findings were contradicted by another study where 83.3 $\%$ of dental students preferred using the circumferential matrix systems (6). Nowadays, the sectional matrix systems are implemented in most of the dental schools in Egypt, both in daily practice and training. These findings confirm the fact that the sectional matricing systems are the most commonly otvorene kontakte s obuhvatnim matričnim sustavima, a nepravilan izlazni profil aproksimalne konture prijavilo je njih 348 (95,8 \%). S druge strane, za sekcijske matrične sustave optimalne kontakte prijavilo je 279 (67\%) stomatologa, tijesne kontakte $109(26 \%)$ i otvorene kontakte 27 (7\%), a pravilan izlazni profilom aproksimalne konture prijavilo 347 $(97,2 \%)$ stomatologa. Postojala je statistički značajna razlika između sekcijskih i obuhvatnih matričnih sustava kad je riječ o tjesnoći aproksimalnih kontaktnih točaka koju su ocijenili stomatolozi $(\mathrm{P}<0,0001)$

\section{Rasprava}

Izrada estetskih aproksimalnih ispuna s pomoću kompozitnih materijala velik je izazov. Hoće li uspjeti stražnji kompozitni ispuni ovisi o vještinama kliničara, karakteristikama materijala i korištenim tehnikama postavljanja (7). U nekoliko istraživanja potvrđeno je da su unaprijed oblikovani sekcijski matrični sustavi kompozitnim ispunima dali optimalne konture i izlazne profile u usporedbi s konvencionalnim ravnim obuhvatnim trakastim matricama $(3,4)$. U nekoliko istraživanja autori su istaknuli da je korištenjem unaprijed oblikovanih sekcijskih matričnih vrpci u kombinaciji sa separacijskim prstenom postignuta optimalna tjesnoća kontakta zahvaljujući interdentalnoj separaciji koju je osigurao prsten tijekom izrade restauracije i konturi matrice koja oponaša prirodne aproksimalne konture i izlazni profil zuba $(3,8-10)$.

$\mathrm{U}$ ovom istraživanju većina stomatologa nije vjerovala da se sustavima obuhvatnih matrica mogu postići dosljedne kontaktne točke. Razlog za manji optimalni aproksimalni kontakt s obuhvatnim matričnim sustavima mogao bi se pripisati nedovoljnom pomicanju susjednih zuba zbog postavljanja interdentalnog kolčića, u usporedbi s interdentalnim separacijskim prstenom i ravnom matričnom trakom. Tijesne kontaktne točke zabilježene su na višoj razini prema okluzalno jer zubni konac nije mogao proći ili je puknuo pri izvlačenju (11). Optimalne kontaktne točke s obuhvatnim matričnim sustavima uglavnom su prijavili iskusniji stomatolozi (dr. sc. i prof. dr. sc.) jer su vjerojatno imali više iskustva s takvim matricama, ali su i oni izlazne profile ocijenili nezadovoljavajućima (12).

Rezultati ove ankete pokazali su da je $74 \%$ stomatologa preferiralo sekcijske matrične sustava, a $63 \%$ smatralo je da su oni sustavi jednostavniji za primjenu u usporedbi s obuhvatnim matričnim sustavima. To se može pripisati prebacivanju $s$ amalgamskih ispuna koji su se nekada postavljali na kompozitne ispune zbog estetskih zahtjeva pacijenata i unaprjeđenja fizičkih i mehaničkih svojstava kompozita, što zahtijeva edukaciju o novim tehnikama i restaurativnim postupcima. No ovim nalazima proturječilo je drugo istraživanje u kojemu je 83,3\% studenata stomatologije preferiralo upotrebu matričnih sustava (6). Danas se sustavi sekcijskih matrica primjenjuju na većini stomatoloških fakulteta u Egiptu u svakodnevnoj praksi i edukaciji. Ta otkrića potvrđuju činjenicu da egipatski stomatolozi najčešće upotrebljavaju sekcijske matrice za postizanje konzistentnih i pouzdanih kontaktnih točaka na stražnjim aproksimalnim kompozitnim ispunima. 


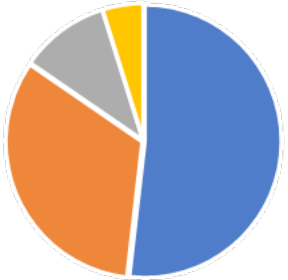

- $\operatorname{BDS}(52 \%, \mathrm{n}=215)$

MSc $(33 \%, \mathrm{n}=136)$

DDSc/PhD (10\%, $\mathrm{n}=44)$

Egyptian Board (5\%, n=20)

Highest academic degree achieved

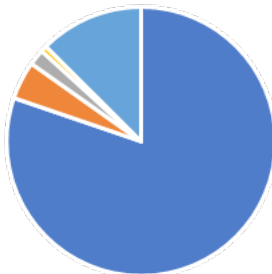

- Tofflemire $(80.2 \%, 333)$

Ivory no 2 (4.6\%, $\mathrm{n}=19)$

- Ivory no $8(2 \%, \mathrm{n}=8)$

(1) Automatrix $(0.7 \%, \mathrm{n}=3)$

Do not use $(12.5 \%, \mathrm{n}=52)$

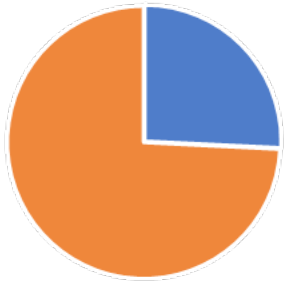

- Circumferential $(26 \%, \mathrm{n}=107)$

- Sectional $(74 \%, \mathrm{n}=308)$

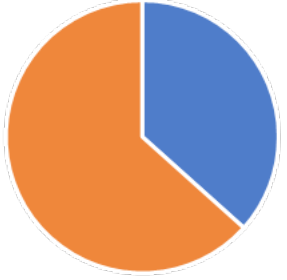

Circumferential $(37 \%, 152)$

- Sectional (63\%, 263)

Easiest matricing technique

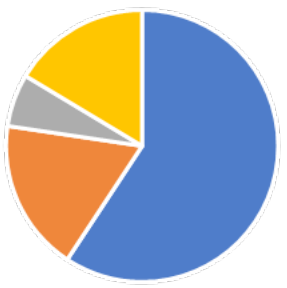

- Wooden $(59 \%, \mathrm{n}=246)$

Diamond $(18 \%, \mathrm{n}=75)$

Elastic $(6 \%, \mathrm{n}=26)$

DPastic $(17 \%, \mathrm{n}=68)$

Torvm saddle $(1 \%, \mathrm{n}=4)$

- Composi-Tight $(20.7 \%, \mathrm{n}=86$ )

- Palodent V3 (15.2\%, n=63)

- Ena ring $(2.9 \%, \mathrm{n}=12)$

- Twin ring $(0.7 \%, \mathrm{n}=3)$

- Do not use $(13.9 \%, \mathrm{n}=58)$

\section{Types of circumferential matrix \\ Types of sectional matrix systems} systems

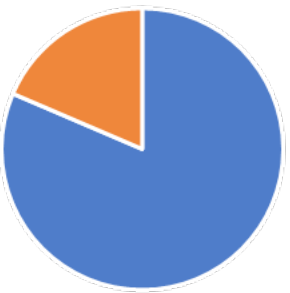

-Yes $(81 \%, \mathrm{n}=338)$ No $(19 \%, \mathrm{n}=77)$

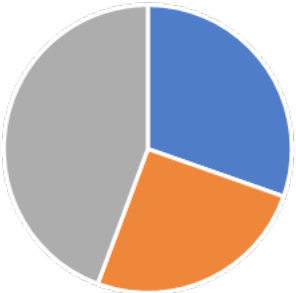

- Optimum (31\%, n=126)

- Tight $(25 \%, \mathrm{n}=105)$

- Improper (95.8\%, $\mathrm{n}=348)$

- Open (44\%, n=184)

Assessment of proximal contacts after restoration
Titghtness of proximal contacts produced by circumferential matricing systems
Emergence profile of circumferential matrix

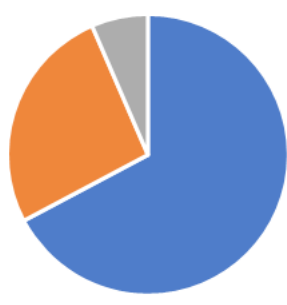

- Optimum (67\%, n=279)

- Tight (26\%, n=109)

- Open $(7 \%, \mathrm{n}=27)$

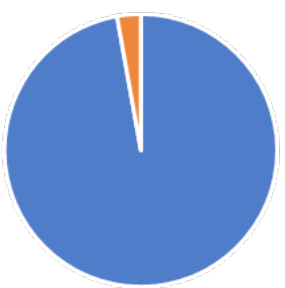

- Proper $(97.2 \%, 347)$

Improper $(2.8 \%, 10)$
Figure 1 Pie charts denoting results of the questionnaire

Slika 1. Tortni grafikoni s rezultatima upitnika 
used systems by Egyptian dentists to achieve consistent and reliable contact points of posterior proximal resin composite restorations.

Among the circumferential matrix systems in the current survey, the Tofflemire matrix holder and bands were most commonly used $(80.2 \%, n=333)$. In most of dental schools in Egypt, this system is most commonly used in the pre-clinical simulation laboratory course (6). The circumferential matrix systems were conventionally used for proximal restorations (13) and the regression in its use was due to the fact that the sectional matrix systems proved to produce anatomically optimal contact points $(7,14-16)$. Among the sectional matrix systems, 54\% of Egyptian dentists mainly used matrix systems manufactured by TOR VM (Moscow, Russia) because they are relatively cheap and abundant in the Egyptian dental market with a wide variety of separation rings and matrices. Twenty percent of dentists in the current study used Composi-tight (Garrison Dental Solutions, Spring Lake, Michigan, USA) while $15.2 \%$ of them used the Palodent V3 system (Dentsply Sirona, Konstanz, Germany). Both systems have proven to have reliable reproduction and consistent proximal contact and contour, but they are expensive.

Wooden and diamond wedges were the most commonly used during the placement of posterior restorations. Many dentists preferred to use wooden wedges since they are cheap and can expand when exposed to oral fluids, which helps in interdental separation and adaption of the matrix band gingivally (17). However, when wooden wedges absorb fluids they become weak and flexible, and they adapt only to the natural anatomic proximal contour, resulting in less interdental separation (14). On the other hand, compared to wooden wedges, diamond wedges need less effort during placement. The diamond shaped cut-out in the tip collapses upon placement, facilitating its insertion inside the embrasure. The diamond wedge then springs open once inside the embrasure, producing a tight gingival seal and preventing the wedge from getting out (18).

In a previous survey investigating into the range of techniques used by UK general dental practitioners when placing posterior composites, 155 dentists (61\%) used the circumferential metal matrix system and a wooden wedge, 74 dentists $(29 \%)$ used the transparent matrix system, and only 25 dentists (10\%) used the sectional metal matrix and a wooden wedge. These results were obtained 11 years ago when the use of the sectional matrix systems was not universally implemented in evidence-based guidelines and amalgam restorations were preferred over the posterior composite restorations (16).

The sectional matrix systems are technique sensitive due to rounded contours and thin thickness of matrices, which can cause a depression or bending in the matrix material during placement, rendering it unusable (6). Previous studies observed statistically significant differences in contact tightness with different circumferential compared to the sectional matrix systems $(6,7,12)$. In the current study, the dentists reported that the sectional matrix systems produced optimum contact points with proper emergence profiles. This was in agreement with another study (4) where sectional matrices combined with separation rings resulted in increased contact
Među obuhvatnim matričnim sustavima u ovom istraživanju najčešće su korišteni držač i trake Tofflemireove matrice $(80,2 \%, \mathrm{n}=333)$. Na većini stomatoloških fakulteta u Egiptu taj se sustav najčešće upotrebljava na pretkliničkim vježbama (6). Sustavi obuhvatnih matrica konvencionalno su se upotrebljavali za aproksimalne ispune (13), a smanjenje njihove uporabe rezultat je spoznaje da sekcijski matrični sustavi proizvode anatomski optimalne kontaktne točke $(7,14-16)$. Od sekcijskih matričnih sustava $54 \%$ egipatskih stomatologa uglavnom je upotrebljavalo matrične sustave proizvođača TOR VM (Moskva, Rusija) jer su razmjerno jeftini i mogu se nabaviti na egipatskom dentalnom tržištu u širokom rasponu separacijskih prstenova i matrica. Dvadeset posto stomatologa u ovom istraživanju upotrebljavalo je sustav Composi-tight (Garrison Dental Solutions, Spring Lake, Michigan, SAD), a 15,2 \% sustav Palodent V3 (Dentsply Sirona, Konstanz, Njemačka). Oba sustava pokazala su se pouzdanima u rekonstrukciji kontaktne točke i kontura, ali su skupi.

Pri izradi stražnjih ispuna najčešće su se upotrebljavali drveni i dijamantni interdentani kolčići. Mnogi su stomatolozi radije odabrali drvene kolčiće jer su jeftini i mogu se širiti ako su izloženi oralnim tekućinama, što pomaže u interdentalnom razdvajanju i prilagodbi matrice gingivno (17). No kada drveni kolčići apsorbiraju tekućinu postaju slabi i fleksibilni te se prilagođavaju samo prirodnoj anatomskoj aproksimalnoj konturi, što rezultira manjom separacijom (14). S druge strane, u usporedbi s drvenim kolčićima, za dijamantne treba manje napora tijekom postavljanja. Rez u obliku dijamanta na vrhu kolabira nakon postavljanja i olakšava njegovo umetanje. Dijamantni kolčić otvara se unutar interdentalnog prostora te stvara čvrstu gingivnu barijeru i sprječava ispadanje kolčića (18).

$\mathrm{U}$ jednom istraživanju u kojemu je analiziran niz tehnika koje su britanski stomatolozi primjenjivali pri izradi stražnjih kompozitnih ispuna, 155 (61\%) stomatologa upotrebljavalo je obuhvatni metalni matrični sustav i drveni kolčić, $74(29 \%)$ koristilo se prozirnim matričnim sustavom, a samo 25 (10\%) upotrebljavalo je sekcijsku metalnu matricu i drveni kolčić. Ta otkrića datiraju od prije 11 godina kada se sekcijski matrični sustavi nisu univerzalno primjenjivali, a na stražnjim zubima češće su se izrađivali amalgamski ispuni od kompozitnih (16).

Sekcijski matrični sustavi osjetljivi su na proceduralne pogreške zbog njihovih zaobljenih kontura i male debljine, što može prouzročiti udubljenje ili savijanje materijala tijekom postavljanja, pa matricu čini neupotrebljivom (6). U dosadašnjim istraživanjima autori su istaknuli statistički značajne razlike u tjesnoći kontakata $s$ različitim obuhvatnim matričnim sustavima u usporedbi sa sekcijskima $(6,7,12)$. U ovom istraživanju stomatolozi su naveli da su sekcijski matrični sustavi stvorili optimalne kontaktne točke s pravilnim izlaznim profilima. To je u skladu s rezultatima jednoga drugog istraživanja (4) u kojem su sekcijske matrice u kombinaciji sa separacijskim prstenovima rezultirale povećanom tjesnoćom kontakta, a upotrebom obuhvatne matrice smanjena je tjesnoća kontakta (7).

U ovoj anketi minimalni broj ispuna koje su ocjenjivali stomatolozi koji upotrebljavaju sekcijske matrične sustave 
tightness, while the use of a circumferential matrix resulted in decreased contact tightness. (7).

In the current survey, the dentists using the sectional matrix systems reported that a minimal number of restorations exhibited open or tight proximal contact. This can be explained by the fact that the separation ring was not effective in separating the teeth, or the clinicians were unable to place the matrix system effectively. The dentists reported that even open or tight contacts produced with sectional matrix systems had proper emergence profiles (12).

\section{Conclusions}

The results of this survey have shown that Egyptian dentists preferred using the sectional matrix systems. Optimum contact points were highly associated with the sectional matrix systems. However, poor (open and tight) contacts were highly associated with the circumferential matrix systems.

\section{Clinical recommendations}

Various restorative techniques have been recommended to reproduce and recreate optimum proximal contact points and contours for compound proximal restorations. It is advocated to embrace the use of sectional matrix systems accompanied with interdental separation rings in daily practice for all dental practitioners. Undergraduate dental students and fresh graduates should be qualified for using the sectional matrix systems to provide the finest dental health services for patients.

\section{Conflict of interest}

The author declares that there is no conflict of interest.

\section{Author's contribution}

O.O.S. - Concepts, design, definition of intellectual content, literature search, data acquisition, data analysis, statistical analysis, sample size calculation, manuscript preparation, manuscript editing, manuscript review and guarantor. imao je otvoren ili pretijesan aproksimalni kontakt. Razlog je vjerojatno to što separacijski prsten nije bio učinkovit u odvajanju zuba ili kliničar nije mogao dobro postaviti matrični sustav. Stomatolozi su izvijestili da su čak i otvoreni ili tijesni kontakti proizvedeni sa sekcijskim matričnim sustavima imali odgovarajuće izlazne profile (12).

\section{Zaključci}

Rezultati ovog istraživanja pokazali su da egipatski stomatolozi uglavnom upotrebljavaju sekcijske matrične sustave. Optimalne kontaktne točke češce su bile povezane sa sustavima sekcijskih matrica. $S$ druge strane, loši (otvoreni i tijesni) kontakti češće su bili povezani s obuhvatnim matričnim sustavima.

\section{Kliničke preporuke}

Preporučene su razne restaurativne tehnike za rekonstrukciju optimalnih aproksimalnih kontaktnih točaka i kontura s kompozitnim ispunima. Rezultati govore u korist primjene sekcijskih matričnih sustava u kombinaciji $s$ interdentalnim separacijskim prstenovima u svakodnevnoj praksi za sve kliničare. Studenti dodiplomskog studija stomatologije i mladi doktori trebali bi znati služiti se sekcijskim matričnim sustavima kako bi svojim pacijentima mogli pružiti najkvalitetniju zdravstvenu uslugu.

\section{Sukob interesa}

Autor nije bio u sukobu interesa.

\section{Doprinos autora}

O. O. S. - koncepti, dizajn, definicija intelektualnog sadržaja, pretraživanje literature, prikupljanje podataka, analiza podataka, statistička analiza, izračun veličine uzorka, priprema rukopisa, uređivanje rukopisa, pregled rukopisa i jamac.

\section{Sažetak}

Svrha rada: Željelo se istražiti sustave interdentalnih matrica kojima se koriste egipatski stomatolozi i procijeniti njihov utjecaj na reprodukciju optimalnih aproksimalnih kontakata kod stražnjih aproksimalnih kompozitnih ispuna. Materijal i metode: Izrađen je online upitnik koji je e-poštom i putem platformi društvenih medija poslan na adrese 785 stomatologa. $U$ istraživanju su sudionici odgovarali na sljedeća pitanja: koji im je najviši postignuti akademski stupanj i koliko iskustva imaju, koje sustave interdentalnih matrica upotrebljavaju, koje marke sustava matrica odabiru, kakva im je procjena aproksimalnih kontaktnih točaka i procjena kontaktnih točaka koje su rekonstruirali te kako procjenjuju izlazni profil restauracije. Rezultati: $U$ istraživanju je ukupno sudjelovalo 415 stomatologa (stopa odgovora 52,8 \%). Od toga je njih 308 (74\%) preferiralo korištenje sekcijskoga matričnog sustava, a 107 (26\%) je radije odabiralo obuhvatni matrični sustav. Stotinu dvadeset i šest (31 $\%)$ stomatologa navelo je da obuhvatni matrični sustav reproducira optimalne kontakte, 105 (25\%) je prijavilo pretijesne kontakte, a 184 (44\%) otvorene kontakte. Za sekcijske matrične sustave optimalne kontakte navelo je 279 kliničara (67\%), tijesne kontakte njih 109 (26\%), a otvorene kontakte 27 (7\%). Postojala je statistički značajna razlika između sekcijskih matričnih sustava i obuhvatnih sustava s obzirom na tjesnoću aproksimalnih kontaktnih točaka $(P<0,0001)$. Zaključak: Egipatski stomatolozi najčešće se koriste sekcijskim matričnim sustavima. Istraživanje je pokazalo da su optimalne kontaktne točke u velikoj mjeri povezane sa sekcijskim matričnim sustavima, a loši (otvoreni i tijesni) kontakti uglavnom sa sustavima obuhvatnih matrica.
Zaprimljen: 3. kolovoza 2020. Prihvaćen: 2. studenoga 2020.

Adresa za dopisivanje Omar Osama Shaalan PhD Faculty of Dentistry Conservative Dentistry Department Cairo University, Cairo, Egypt. 35 Mohamad Farid Street El Hay El Motamayz, tel: (002)01122665661, faks: (002) 38831188, omar.shaalan@dentistry.cu.edu.eg

Ključne riječi obuhvatna matrica, II. razred, FDI, trakasta matrica, aproksimalno, kompozitni materijal 


\section{References}

1. Jo D-W, Kwon M-J, Kim J-H, Kim Y-K, Yi Y-J. Evaluation of adjacent tooth displacement in the posterior implant restoration with proximal contact loss by superimposition of digital models. J Adv Prosthodont. 2019 Apr;11(2):88-94.

2. Khan FR, Umer F, Rahman M. Comparison of proximal contact and contours of premolars restored with composite restoration using circumferential matrix band with and without separation ring: A randomized clinical trial. Int J Prosthodont Restor Dent. 2013;3(1):7-13.

3. Saber MH, Loomans AC, Zohairy A El, Dörfer CE, El-Badrawy W. Evaluation of proximal contact tightness of Class II resin composite restorations. Oper Dent. Jan-Feb 2010;35(1):37-43.

4. Wirsching E, Loomans BAC, Klaiber B, Dörfer CE. Influence of matrix systems on proximal contact tightness of 2-and 3-surface posterior composite restorations in vivo. J Dent. 2011 May;39(5):38690.

5. Brackett MG, Contreras S, Contreras R, Brackett WW. Restoration of proximal contact in direct class II resin composites. Oper Dent. Jan-Feb 2006;31(1):155-6.

6. Ahmad MZ, Gaikwad RN, Arjumand B. Comparison of two different matrix band systems in restoring two surface cavities in posterior teeth done by senior undergraduate students at Qassim University, Saudi Arabia: A randomized controlled clinical trial. Indian J Dent Res. Jul-Aug 2018;29(4):459-464.

7. Loomans BAC, Opdam NJM, Roeters FJM, Bronkhorst EM, Burgersdijk RCW, Dörfer CE. A randomized clinical trial on proximal contacts of posterior composites. J Dent. 2006 Apr;34(4):292-7.

8. Demarco FF, Cenci MS, Lima FG, Donassollo TA, de Almeida André D, Leida FL. Class II composite restorations with metallic and translucent matrices: 2-year follow-up findings. J Dent. 2007 Mar;35(3):231-7.
9. Prakki A, Otávio Chalup Saad J, Rodrigues JR. Clinical evaluation of proximal contacts of Class II esthetic direct restorations. Quintessence Int (Berl). 2004;35(10):785-9.

10. Deepak S, Nivedhitha MS. Proximal contact tightness between two different restorative materials-An in vitro study. J Adv Pharm Educ Res 2017;7(2):153-156.

11. Loomans BAC, Opdam NJM, Roeters FJM, Bronkhorst EM, Burgersdijk RCW. Comparison of proximal contacts of Class II resin composite restorations in vitro. Oper Dent. Nov-Dec 2006;31(6):68893.

12. Kampouropoulos D, Paximada C, Loukidis M, Kakaboura A. The influence of matrix type on the proximal contact in Class II resin composite restorations. Oper Dent. 2010;35(4):454-62.

13. van der Vyver PJ. Posterior composite resin restorations. Part 3. Matrix systems. SADJ. 2002 Jun;57(6):221-6.

14. Loomans BAC, Opdam NJM, Roeters EM, Bronkhorst FJM, Dörfer CE. A Clinical Study on Interdental Separation Techniques. Oper Dent. May-Jun 2007;32(3):207-11.

15. Peumans M, Van Meerbeek B, Asscherickx K, Simon S, Abe Y, Lambrechts P, et al. Do condensable composites help to achieve better proximal contacts? Dent Mater. 2001 Nov;17(6):533-41.

16. Gilmour ASM, Latif M, Addy LD. Placement of posterior composite restorations in United Kingdom dental practices : techniques, problems, and attitudes. Int Dent J. 2009 Jun;59(3):148-54.

17. Gupta R, Hegde J, Prakash V, Srirekha A - editors. In: Concise Conservative Dentistry and Endodontics. Elsevier Health Sciences; 2019.

18. De La Peña VA, García RP, García RP. Sectional matrix: Step-bystep directions for their clinical use. Br Dent J. 2016;220(1):11-4. 